\title{
Mechanism of water/ion exchange at a protein surface: a weakly bound chloride in Helicobacter pylori apoflavodoxin.
}

\author{
Juan J. Galano-Frutos ${ }^{\text {a,b }}$, M. Carmen Morón ${ }^{\text {c,d }}$ and Javier Sancho ${ }^{\text {a,b }}$ \\ a Departamento de Bioquímica y Biología Molecular y Celular, Facultad de Ciencias, \\ Universidad de Zaragoza, Pedro Cerbuna 12, 50009, Zaragoza, Spain. \\ ${ }^{\mathrm{b}}$ Biocomputation and Complex Systems Physics Institute (BIFI). Joint Unit BIFI-IQFR (CSIC). \\ Edificio I+D, Mariano Esquillor, 50018, Zaragoza, Spain. \\ ${ }^{\mathrm{c}}$ Instituto de Ciencia de Materiales de Aragón (ICMA), Consejo Superior de Investigaciones \\ Científicas-Universidad de Zaragoza, Pedro Cerbuna 12, 50009, Zaragoza, Spain. \\ d Departamento de Física de la Materia Condensada, Facultad de Ciencias, Universidad de \\ Zaragoza, Pedro Cerbuna 12, 50009, Zaragoza, Spain.
}

\section{Correspondence to:}

Javier Sancho; e-mail: jsancho@unizar.es;

Phone: (+34) 976 761286;

Fax: (+34) 976762123. 


\section{ABSTRACT}

Binding/unbinding of small ligands, such as ions, to/from proteins influence biochemical processes such as protein folding, enzyme catalysis or protein/ligand recognition. We have investigated the mechanism of choride/water exchange at a protein surface (that of the apoflavodoxin from Helicobacter pylori) using classical all-atom molecular dynamics simulations. They reveal a variety of chloride exit routes and residence times, the later related with specific coordination modes of the anion. The role of solvent molecules in the mechanism of chloride unbinding has been studied in detail. We see no temporary increases in chloride coordination along the release process. Instead, coordination of new water molecules takes place in most cases after the chloride/protein atom release event has begun. Moreover, the distribution function of water entrance events into the first chloride solvation shell peaks after chloride protein atom dissociation events. All these observations together seem to indicate that water molecules simply fill the vacancies left by the previously coordinating protein residues. We thus propose a step-by-step dissociation pathway in which protein/chloride interactions gradually break down before new water molecules progressively fill the vacant positions left by protein atoms. As observed for other systems, waters associated to bound chloride or to protein atoms have longer residence times that those bound to the free anion. The implications of the exchange mechanism proposed for the binding of the FMN (Flavin Mononucleotide) protein cofactor are discussed. 


\section{INTRODUCTION}

Proteins are typically surrounded by and interact with other molecules, including solvent and dissolved ions. Binding and unbinding of small ligands from proteins govern the strength of the complexes formed and bear on important biochemical issues such as protein stability or enzyme catalysis. ${ }^{1,2}$ On the practical side, they bear on the field of drug design where the importance of controlling not just the affinity of a drug, but also its average residence time on a given target is widely recognized. ${ }^{3}$

Since then up to nowadays, many theoretical and experimental studies dealing with the dynamic of biological water have been published, ${ }^{5-14}$ and there is a consensus that the behaviour of these water molecules is different from the bulk in several ways, e.g. polar properties, density, residence times, or the way they cluster. ${ }^{5,6,13,14}$ The dynamics of biological water has been detected by a series of experimental techniques and showed to display an almost bulk-water like ultrafast component $(\sim 1 \mathrm{ps})$ and a surprisingly ultraslow contribution at the $100-1000$ ps time scale. $^{5,9,15}$ The ultrafast component arises from an extended hydrogen bond network while the ultraslow derives from the binding of water to the biomolecule. Biological water, ${ }^{16}$ and solvent in general, play a fundamental role in mediating many biological processes ${ }^{17}$ such as protein-protein and protein-DNA molecular recognition, ${ }^{18-20}$ protein folding, ${ }^{21-24}$ enzymatic catalysis, ${ }^{2}$ electron transfer ${ }^{25}$ or proton transfer. ${ }^{26}$ On the other hand, many proteins are able to bind ions dissolved in water. Bound cations often act as cofactors, either stabilizing the protein (structural role) or being involved in enzymatic reactions (functional role). ${ }^{27}$ Fewer examples of specific roles for anions in biological systems are known, ${ }^{28-34}$ which may relate to the lower charge density of anions relative to cations probably contributing to weaker anion/protein interactions.

Protein engineering ${ }^{35}$ is a useful approach to investigate structural aspects related to the 
binding mechanisms of small ligands to proteins. ${ }^{36-39}$ Anion/protein binding mechanisms for a few anion-binder proteins has been studied using site-directed mutagenesis in the last two decades. ${ }^{28-30,32,33}$ While this kind of approaches is helpful and may provide important clues, it is not very appropriate to deal with aspects such as flexibility or dynamics. Moreover, gathering enough structural information on the activated complexes from experimental transition state analysis of binding kinetics, typically fails due to the small number of appropriate deletion mutations that can be engineered in key binding residues. ${ }^{36,37}$ Alternative approaches increasingly rely on Molecular Dynamics (MD) methods to investigate these issues. In this respect, techniques capable of effectively sampling energy landscapes and speeding up the analysis of the kinetics taking place on the microsecond or higher time scales (such as the aforementioned binding or unbinding of small ligands to/from proteins) have been implemented. ${ }^{40,41} \mathrm{MD}$ allows estimating fairly accurately a variety of atomistic and thermodynamic parameters, ${ }^{42-44}$ providing a detailed description of the behaviour of macromolecules submerged in solvent.

For some time we have been investigating protein/ligand binding mechanisms using flavodoxins. ${ }^{36,37,39,45-48}$ These electron-transfer proteins constitute simple and convenient models for investigating protein/ligand recognition and other basic molecular issues such as protein folding and stability. ${ }^{49-52}$ In addition, flavodoxins have been revealed as promising drug targets to develop novel antibiotics, ${ }^{53-56}$ particularly the flavodoxin from Helicobacter pylori $(\mathrm{Hp}$-Fld $) .{ }^{57}$ In this flavodoxin, the apoprotein ${ }^{48}$ binds a chloride anion at the site where the phosphate group of the FMN (Flavin Mononucleotide) cofactor appears bound in the functional holoprotein. Similarly, the apoflavodoxin from Anabaena PCC 7119 carries a sulfate anion at the equivalent $\operatorname{site}^{47}$ (see Fig. 1). Driven by our interest in the mechanism of anion/water exchange at protein surfaces and in its implications for the binding of biological cofactors such as FMN, we have run 
exploratory classical MD simulations of these two anion-bound apoflavodoxins. In particular, we wanted to find out whether the spontaneous release of either the sulfate or the chloride anion could be observed in short simulation times. While the sulfate bound to Anabaena apoflavodoxin was not released in any of such preliminary simulations, the chloride bound to $H p$ apoflavodoxin was early released in most of them. Because this provides a fine opportunity to study the mechanism of ion/water exchange, using MD we have run a total of 60 simulations of $H p$ apoflavodoxin (20 ns long each) and observed many chloride release events from which we have derived the statistics of the process. Two alternative mechanisms for FMN binding to apo $H p$-Fld can be envisioned: 1) FMN attacking its natural binding site and subsequently forcing a supposedly well-coordinated chloride out from the protein, and 2) a weakly coordinated chloride spontaneously leaving its binding site before the FMN cofactor gets bound. A key point here would be to understand whether chloride release must be initiated by solvent molecules coordinating to chloride protein ligands. To clarify this point a detailed statistic analysis of simulations where chloride unbinds has been carried out. Based on it we propose a plausible binding/unbinding mechanism for chloride/Hp-Fld complex formation and discuss on the possible implications for the mechanism of FMN binding to apoflavodoxins.

\section{METHODS}

\section{IIa. Simulation procedure details}

We have performed classical all-atom MD simulations of apo $H p$-Fld in a conveniently neutralized water box. The atomic coordinates of the apo $\mathrm{Hp}$-Fld (wild type) were taken from the Protein Data Bank (PDB code: $2 \mathrm{BMV}){ }^{48}$ Crystallographic waters were eliminated as well as one benzamidine molecule present as an additive in the crystallization buffer and $\mathrm{H}$-bonded to the 
side chain of GLU30 (far away from the active site). However, the chloride anion present in the structure at the site where the phosphate group of FMN appears bound in the holoprotein was kept in its original position. The solvation and neutralization of the system together with the visualization of trajectories were accomplished through packages and plugins of VMD 1.9.1. ${ }^{58,59}$ All simulations were performed using NAMD $2.9^{60}$ in an CPT ensemble. ${ }^{61}$ The CHARMM27 force field with CMAP correction was used. ${ }^{62}$ The analysis of the trajectories was carried out through VMD, and a set of ad hoc TCL and Perl scripts.

After removal of crystallographic waters and the benzamidine molecule, the protein was immersed in a TIP3P explicit water box with dimensions $53.1 \AA$ x $55.8 \AA$ x $58.9 \AA$ (2388 water molecules). Following NAMD standard procedure we adjusted the dimensions of the box to ensure a minimum solvation shell of $7 \AA$ for the outer atoms of the protein. This system was conveniently neutralized by adding 14 sodium counterions in positions close to negatively charged residues. At this point the system contains 9598 atoms.

Before the production phase, the system was prepared as follows. Firstly, a steepest-descent minimization step of $5000 \mathrm{fs}$, where both protein atoms and the chloride anion were fixed, was conducted. Then, the system was equilibrated by performing CPT dynamics of $1 \mathrm{~ns}$ in order to relax water/protein interactions. Afterwards, a slow release of the protein atoms, by imposing decreasing elastic restraints, was carried out. In this step, a 10 sub-steps loop of 5000 fs each one was performed starting with a constraint scaling factor of 20 until the constraint was totally removed; a basic force constant of $1 \mathrm{kcal} / \mathrm{mol} / \AA^{2}$ was used. Later, a gradual heating up to $310 \mathrm{~K}$ was performed through a temperature ramp consisting of a 30 sub-steps loop of $0.1 \mathrm{~ns}$ each one. Finally, the system was equilibrated for $2 \mathrm{~ns}$ with all the constraints released. At this stage different seed random numbers were used in order to obtain 50 simulations (replicas). The main 
observable differences found between these replicas at the end of the preparation stages -the initial time of simulation, i.e. $\mathrm{t}=0$ - refer to the chloride 'status' and conformational aspects related to its binding to the apoprotein, as will be discussed below (section III).

The structural models obtained at the end of the preparation phases were used as initial systems for the production phase of $20 \mathrm{~ns}$. In some of the 50 replicas that production phase was extended up to $100 \mathrm{~ns}$ and the temperature was increased by means of a temperature ramp of 1 $\mathrm{K} / \mathrm{ns}$ (discussed below in section III). All the MD simulations that are presented in this work were performed in the CPT ensemble. Thus the pressure and temperature were held constant at 1 atm and $310 \mathrm{~K}$ using the Nose-Hoover Langevin piston ${ }^{61,63}$ and the Langevin thermostat $^{61}$ respectively, except in those cases where the temperature was increased. A cutoff distance of 14 $\AA$ was used for the van der Waals interactions with a switching function from $12 \AA$. Periodic boundary conditions (PBC) were applied to avoid the edge effect, and long-range electrostatic interactions were modeled using the Particle-Mesh Ewald (PME) method. ${ }^{64}$ An integration time of one femtosecond ( $1 \mathrm{fs}$ ) was used for all simulations. The production runs were saved every 5 ps for later analysis.

Finally the systems were checked to assess proper equilibration. Thus, a RMSD (root mean square deviation) analysis for each of the 50 trajectories was done in order to determine the stability of the protein along the production phase, and how different from the initial apo $\mathrm{Hp}$-Fld structure (PDB file) the protein becomes. A RMSD plot, which is representative of the whole set of RMSD analyses, is depicted in Fig. S1. The RMSDs never exceeded $1.5 \AA$, and no visible distortions on the secondary and tertiary protein structures were detected.

In order to perform a finer analysis we have also run 10 additional high-resolution simulations within the interval where chloride release is detected (see sections IIIc and IIId). In this case the 
production runs were saved every 50 femtoseconds for later analyses.

\section{IIb. Radial distribution functions for Chloride-Protein and Chloride-Water pairs}

We have calculated the radial distribution functions, $g(r)$, for chloride-protein and chloride-water pairs in order to determine appropriate cutoff distances (see Fig. 2). As a result, a hydrogen bond is considered to be formed between a chloride anion and a side chain-nitrogen atom of the protein if the distance between those two atoms is smaller than $3.9 \AA$ (see Fig. 2) and the DonorHydrogenAcceptor angle is higher than $110^{\circ}{ }^{65}$ In the case of the $\mathrm{Cl}-\mathrm{O}$ pairs, when $\mathrm{O}$ represents both the oxygen of a water molecule or the sidechain-oxygen of the protein, the criteria for the angle is maintained but the maximum allowed distance $\mathrm{Cl}-\mathrm{O}$ is taken as $3.7 \AA$ (see Fig. 2). Those distances are in good agreement with experimental and simulated data for chloride anion interactions with peptides and water. ${ }^{66,67}$

\section{IIc. Definitions}

In order to precisely analyse the role of water molecules in the mechanism of chloride release we have defined the following terms that will be used throughout section III:

DCR (definitive chloride release): an event consisting in a irreversible release of the chloride from the protein, which we algorithmically detected when for 5 or more consecutive frames of time the distances between the backbone nitrogen atoms of residues ASP11 and SER12, Cl$\mathrm{N}_{\mathrm{ASP} 11}$ and Cl-N $\mathrm{N}_{\mathrm{SER} 12}$ are larger than $7 \AA$. We have found that these two residues are, in general, the last ones in being released from the anion among those six residues that conform the chloride binding site in $H p$-Fld (see Fig. 3). Concerning the choice of that cutoff ( $7 \AA$ ), we have followed the criterion that a chloride ion could be regarded as definitely released from the apoprotein if there is at least one water molecule between the anion and the biomolecule. If a water molecule 
mediates the interaction between both entities, that situation corresponds to a chloride-protein distance ranging between 4 to $7 \AA{ }^{66}$ depending on the particular structural arrangement between the three entities involved: anion, water molecule and polar atom of the biomolecule. On that basis we have considered reasonable the choice of the above referred cutoff of $7 \AA$ for the definition of a definitive chloride release event. Within this scenario we have also considered the moment in which the chloride-protein interaction starts to weaken leading to the definitive chloride release. Thus we have defined FIDCR as the first retrospective instant in which one of the $\mathrm{Cl}-\mathrm{N}_{\mathrm{ASP} 11}$ or $\mathrm{Cl}-\mathrm{N}_{\mathrm{SER} 12}$ distances are larger than $3.5 \AA$ during the above referred release. Since we are interested in the moment in which the chloride-protein interaction starts to weaken, we have preferred to choose a cutoff a bit smaller, $3.5 \AA$, than that corresponding to the maximum radius of the first hydration shell (see Cl-N $\mathrm{N}_{\text {protein }}$ radial distribution functions in Fig. 2).

WE (water entrance): is defined as a steady entry of one water molecule into the first solvation shell of the chloride, forming an H-bond. This event is accounted once a water molecule simultaneously meets the following two requirements: 1) the chloride/water H-bond distance steadily (for 5 or more consecutive frames, i.e. 250 fs) stays below $3.7 \AA$ (see section IIb and Fig. 2) and, 2) the average of the chloride/water H-bond angle for these 5 consecutive frames is higher than 110 degrees. ${ }^{65}$ In terms of time, FIWE is defined as the first retrospective instant in which such water molecule steadily enters the first solvation shell of the chloride.

PAR (protein atom release): is defined as an individual release of a polar atom of the protein, either $\mathrm{N}$ or $\mathrm{O}$, from the chloride coordination sphere. Thus, PAR events are accounted whenever a polar atom of the sub-pocket 1 (see Fig. 3 and Fig. 4), steadily overcomes (for 5 or more consecutive frames, i.e. $250 \mathrm{fs}$ ) the cutoff distance of $4.5 \AA$. This smaller distance in comparison with the $7 \AA$ used for establishing the chloride definitive exit (see DCR definition above) obeys 
to the fact that, often, temporary non-definitive releases of polar atoms occur. As in FIDCR, we define FIPAR as the first retrospective instant in which the concerned polar atom separates more than $3.5 \AA$ from the chloride during the above referred release.

\section{IId. Residence time analysis of water}

For the residence time analysis of water that is performed in section IIId, cutoff distances of 3.7 $\AA$ and $4.5 \AA$ were established to define the first solvation shell around the chloride and the first solvation shell around the chloride binding site, respectively. The second cutoff is $\sim 1 \AA$ larger than the maximum H-bond distance for protein/water interactions. ${ }^{66}$ Such a cutoff was preferred since the chloride binding site is defined as the center of mass of the backbone nitrogens from residues THR10 and GLY13. These atoms remain quite static approximately facing each other during the simulations, so that this point mimics well the position of the chloride inside this pocket along the trajectories.

\section{IIe. Peak-shape function: fit of WE histograms}

In order to fit the WE histograms, obtained by using different FIPAR cutoff distances in section IIIc, an asymmetric pseudo-Voigt function was used: ${ }^{68}$

$y=y_{0}+A\left[\mu\left(\frac{2}{\pi} \cdot \frac{w(t)}{4\left(t-t_{c}\right)^{2}+w(t)^{2}}\right)+(1-\mu)\left(\frac{2}{w(t)} \sqrt{\ln 2 / \pi} \cdot e^{-4 \ln 2\left(\frac{t-t_{c}}{w(t)}\right)^{2}}\right)\right]$

where:

$w(t)=\frac{2 w_{0}}{1+\exp \left[a\left(t-t_{c}\right)\right]}$

The pseudo-Voigt function is a popular mathematical expression for modeling peak shapes. ${ }^{69}$ That function is a linear combination of a Gaussian and a Lorentzian curves (see equation (1)). ${ }^{68}$ The sigmoidal expression defined in equation (2) confers asymmetry to equation (1). When $a$ 
equals zero, then $w(t)=w_{0}$ and equation (1) reduces to the standard symmetric Pseudo-Voigt function. The parameter $A$ defines the area under the peak, $y_{o}$ represents the background, $w_{0}$ stands for the full width at half maximum (FWHM) corresponding to the symmetric curve, $t_{\mathrm{c}}$ defines the maximum of the peak, and $\mu$ is a combinatory coefficient constrained between 0 and 1 which indicates the fraction of the Gaussian $(\mu \sim 0)$ and Lorentzian $(\mu \sim 1)$ curves that has been used.

\section{RESULTS AND DISCUSSION}

\section{IIIa. Chloride release times and exit routes}

Fifty 20 ns-long MD simulations of $H p$ apoflavodoxin were initially carried out using different seed numbers to distribute the initial atomic velocities in each simulation (see section IIa). In 90 $\%$ of those simulations a chloride ion release from the protein was detected at different stages of the simulations (see Fig. 4c).

Clear differences were observed in the anion exit routes. In some simulations, the chloride was readily released into the bulk water while, in others, it was released in less expeditious ways following a sequential process in which it first became unbound from some residues but still remained bound to some others for a time. A similar behaviour has been reported for sodium and chloride ions bound to the small globular protein S6 of the bacterial ribosome. ${ }^{70}$ To explain the different exit routes observed, the FMN binding pocket can be divided into three sub-pockets (see Fig. 4a). The interaction of chloride with these three sub-pockets along the release trajectories has been quantified from the interaction forces (van der Waals + electrostatic) established between the anion and the residues in each sub-pocket. Forces-vs.-time plots of representative examples of direct chloride release into the bulk, or of chloride transfer from the initial location in sub-pocket 
1 to either sub-pocket 2 or 3 are shown in Fig. S2. The statistics of the different behaviours indicates that there appears to be no preferential chloride exit route into solvent (see Fig. 4b)

\section{IIIb. Chloride coordination in fast and in slow release trajectories}

In order to analyse the influence of the chloride coordination on the speed of anion release, representative examples of chloride coordination in delayed releases (Fig. 3a) or early-release trajectories (Fig. 3b) are compared to the situation observed in the crystal structure (Fig. 3c). All distances below the maximum commonly accepted for H-bonds, $4.0 \AA,^{71,72}$ are shown. The average of anion-polar atoms distances is about $2.5 \AA$ in the first case (see Fig. 3a) but significantly increases to around $3.5 \AA$ in the second and third cases (see Fig. $3 \mathrm{~b}$ and Fig. 3c, respectively). Thus, the coordination in delayed-chloride-release simulations (Fig. 3a) appears tighter than in early-chloride-release (Fig. 3b) and also than in the crystal structure (Fig. 3c), indicating it emerges during the simulation. In those delayed-chloride-release simulations about 5 nitrogens atoms, from both backbone and side-chains, appear bound to the chloride for most of the trajectory, as shown in Fig. 3a. To better understand the influence of the chloride coordination on the speed of release we have computed the number of $\mathrm{H}$-bonds formed by the chloride along the simulations, i.e. 18 simulations where this anion was released in the 20 -ns production stage (group 1) and 5 simulations where it was not released (group 2). The increasing-temperature simulations, i.e. the extended phases of group 2 simulations, were also analysed and termed group 2-ext. Averages of, the number of chloride/nitrogen, chloride/oxygen and chloride/water H-bonds (Cl-N_hb, Cl-O_hb and Cl-W_hb respectively), as well as the total number of H-bonds (Total_hb $=$ Cl-N_hb + Cl-O_hb + Cl-W_hb), were calculated (see Table 1). An H-bond cutoff angle of 110 degrees in all the cases ${ }^{65}$ and specific H-bond cutoff distances for each particular 
pair were used (see section IIb). The point of trajectory where the chloride is considered as definitively released (i.e. the point from which trajectories were divided in 'before' and 'afterward', see Table 1) is that in which the anion has moved away from the backbone nitrogen

of both ASP11 and SER12 by at least $7 \AA$ (see DCR definition in section IIc). It is worth noting that, despite group 2 presents a slightly smaller total coordination degree (6.5) relative to group 1 (7.2), their number of $\mathrm{H}-$ bonds coming from protein, i.e. $\mathrm{Cl}-\mathrm{N} \_$hb plus $\mathrm{Cl}-\mathrm{O} \_$hb $(4.8+0.3=5.1)$, is significantly higher than in group $1(2.4+1.1=3.5)$, which is mainly due to the higher number of H-bond formed from nitrogen atoms (4.8vs. 2.4, respectively).

We further investigated how chloride coordination was changing with the approaching of the definitive chloride unbinding event by carrying out the same analysis but now on 1 ns of trajectory around this point $(0.5 \mathrm{~ns}$ on each side). The two groups considered are now termed group 1A and group 2-extA (see Table 1). The Cl-O_hb values (1.2 for both groups), higher than those in group 1 (1.1) and group 2-ext (0.5), indicate that the weakening of chloride/protein interactions is not due to changes in chloride/oxygen $\mathrm{H}$-bonds. In contrast, the Cl-N_hb values were decreased (1.5 for group 1A and 2.3 for group 2-extA) relative to those in the entire simulations (2.4 for group 1 and 4.1 for group 2-ext), suggesting that the chloride leaves its binding site once the number of coordinated nitrogen atoms is significantly reduced, whatever the underlying cause. Short movies of two trajectories showing chloride release from the apo $H p$-Fld are presented in the Supplemental Material (SM).

\section{IIIc. Solvent role in the mechanism of chloride unbinding from the apoprotein}

The trajectories where the chloride is spontaneously released during the productive phase conducted at $310 \mathrm{~K}$ provide an opportunity to understand the mechanism at atomic level, which 
may offer insights into the role of water in anion exchange by proteins and hence into the role of the latter in cofactor binding processes. ${ }^{36}$ One key point here is the order of events during chloride release that lead to water binding to and to chloride unbinding from the apoprotein. Specifically, we would like to know whether the entry of additional water molecules into the first solvation shell of the coordinated chloride is required to initiate the definitive release of this anion or not. To shed light on this issue we conducted 10 new simulations at a higher resolution (50 femtoseconds, see section IIa) in an appropriate interval where chloride release was observed. The starting ten conformations for these simulations were selected from the 18 productive stages in which the chloride was released so that they were representative of both complexes with a high (4-5) and a lower $(<4)$ number of protein polar atoms coordinating the chloride (see Fig. 3).

We first determined whether the coordination number of chloride increases due to the incorporation of additional water molecules just before chloride release. In such a scenario new water molecules would enter the first solvation shell of the anion thus exceeding its common valence and subsequently promoting the anion release from its original binding site. Plots of the temporal evolutions of chloride/protein, chloride/water and total chloride H-bond numbers around the anion release point (see Fig. 5a) indicate that the total number of H-bonds remains constant during the chloride release and that the number of chloride/water H-bonds seems to increase just immediately after a decrease in the chloride/protein H-bond number takes place. This seems to indicate that water molecules simply fill the vacancies left by the previously coordinating protein residues.

We then analysed the temporal evolutions of the distances between chloride and the main atomic entities involved in the release mechanism, namely, the closest polar atoms of the protein to the chloride -i.e. the backbone and side-chain polar atoms of residues THR10, ASP11, SER12, 
GLY13 and ASN14- and the water molecules that entered the first solvation shell of the chloride close around the anion release instant (see Fig. S3). To interpret those distance graphs it is necessary to previously define meaningful cutoffs that allow setting both the point where chloride begins to be definitively released from the protein (first instant in definitive exit of chloride: FIDCR, see section IIc) and the point in which one water molecule enters the first solvation shell of the chloride (first instant in water entrance: FIWE, see section IIc). One representative analysis of a chloride release trajectory indicating the FIDCR and FIWE points associated with those water molecules entering the coordination sphere of chloride right in that surroundings is shown in Fig. 5b. In overall, this FIDCR/FIWE analysis indicates that the steady entries of new water molecules into the first solvation shell of the chloride (WE events) take place in most cases (8 out of 10) after the starting moment of the definitive chloride release event (FIDCR). In two cases, nevertheless, it is difficult to draw a conclusion because, due to the greater instability of chloride coordination observed in those trajectories, the calculated FIDCR may not correspond with the point where chloride/protein interaction really begins to weaken (see Fig. S3).

Finally, we have carried out a statistical analysis of water entrance (WE) events into the first solvation shell of the chloride in the form of histograms. To this end, we have first defined and identified along the ten high-resolution trajectories FIPAR moments as those time points where a protein polar atom start to release from the chloride, i.e. each and every one of the very first instants where a chloride/protein H-bond has begun to break down (see PAR definition in section IIc). Trajectory fragments with a fixed number of frames close around each identified FIPAR moment were extracted and superimposed together on a common time frame defined by assigning $t=0$ to each FIPAR instant. In this way WE events registered during each of these time intervals can be summed up upon each individual frame of time, giving rise to histograms that 
indicate, on the whole, whether the occurrence of WEs precedes or follows FIPAR events. Fig. 6 shows histograms of the WE events registered in trajectory fragments of 50 frames around FIPARs ( 25 frames on each side). A total of 115 PARs and subsequently FIPARs were identified through the ten analysed trajectories. The histogram on the right in Fig. 6 represents a 'compacted' version where the registered WE events are grouped in five consecutive frames of times. The main goal of this analysis is to determine whether there is an increase in WE events at $t<t_{o}$ (preceding FIPARs), which would suggest that water molecules actively contribute to cause the chloride unbinding event. The obtained histograms do not show any increase in WEs before $t_{o}$. Instead, a mild decrease can be noticed, consistent with the chloride unbinding process not originating in a higher coordination of water molecules to the anion. Registration of FIPAR moments and WEs events displayed in histograms in Fig. 6 is based on cutoff distances of $3.5 \AA$ and $3.7 \AA$ respectively (see section IIc).

Since the choice of the FIPAR cutoff distance may influence the shape of the WE histogram, we have studied this influence by obtaining analogous histograms using cutoff distances for FIPAR registrations of up to $0.2 \AA$ above and below the originally taken cutoff value, i.e. from 3.3 to $3.7 \AA$. Fig. 7 shows 'compacted' histograms for WEs obtained by using cutoff distances in this range, namely, every $0.1 \AA$. Here, all the newly obtained histograms also show no increase in WEs before $t_{o}$ as well as that the maximum peak appears after the FIPARs. In order to mathematically assess such observed behaviours a pseudo-Voigt asymmetric function has been used to fit the WEs distributions (see section IIe). The time position corresponding to the maximum of the peak-shape curve appears skewed into the right of $t_{o}$ in all the analysed cases. These results support the conclusion given through this analysis, and at the same time allow establishing a valid range of cutoff distances for chloride/protein interactions in the case that 
similar analyses be required.

\section{IIId. Water residence times at the chloride anion and its binding site, before and after chloride release}

The residence time $e^{5,6,9,12-14}$ of each water molecule associated to either the chloride anion or to the chloride binding site has been determined along each of the 10 high-resolution simulations. A cutoff distance of $3.7 \AA$ has been used to define the solvation shell of the anion, while a cutoff of $4.5 \AA$ from the center of mass of the backbone nitrogens from residues THR10 and GLY13 defines the first solvation shell of the binding site (see section IId). Graphs in Fig. S4 show the presence of a particular water molecule in a given frame as an asterisk so that the lengths of the lines formed by the asterisks are proportional to the residence times of the water molecules. Residence times as long as hundreds of picoseconds have been reported for sodium and chloride ions bound to the $\mathrm{S} 6$ ribosomal protein. ${ }^{70}$

According to graphs in Fig. S4 no significant differences are found between the residence times of waters in either the chloride first shell or in the chloride binding site in early times of the simulation compared to those immediately before a chloride release event, which can be interpreted as a further evidence of no active role played by solvent molecules in stimulating chloride release. On the other hand, release of the anion affects in a different way to waters in the binding site and waters bound to the chloride. While the former apparently experience no changes in their residence times, those bound to the free anion exchange more rapidly reducing their residence times. Higher residence times, than those reported for bulk water, have been also found for water molecules located in the immediate vicinity of a biomolecule ${ }^{6}$ (biological water, see Introduction section). Thus, waters associated to bound chloride behave like biological water in 
the sense that they exhibit higher residence times than those bound to the free anion which are in faster exchange. These observations are consistent with the ultraslow component of the dynamics reported for biological water near protein surfaces respect to the bulk,,$^{5,6,9,13,14}$ and suggest that attractive forces are exerted by the protein upon those water molecules, which might contribute indirectly to a tighter coordination of the anion. Table S1 summarizes statistics gathered from the previous analyses, which provide further evidence that solvent does not play a relevant role in the chloride unbinding process.

\section{IIIe. Chloride unbinding mechanism from $H p$ apoflavodoxin}

The previous analyses indicate that the total number of chloride H-bonds remains constant during definitive anion release events and that a decrease in chloride/protein H-bonds is immediately followed by an increase in chloride/water H-bonds (both in individual PARs and in definitive chloride release events). Based on this evidence we propose a sequential mechanism for chloride release from $H p$ apoflavodoxin which is illustrated in Fig. 8. Chloride release is initiated by protein/chloride H-bond breaking events (the cause of which is not here investigated) that are followed by opportunistic binding of solvent water molecules without net increase of the total coordination number. The upper path in Fig. 8 envisioning for water molecules a role in destabilizing chloride/protein H-bonds by joining the first coordination shell and increasing the coordination number is not consistent with our analysis. Therefore, the reverse process of chloride binding to the apoprotein would proceed via opportunistic binding by protein atoms to vacant positions in the chloride coordination shell originating from water dissociation events not forced by the protein. In order to illustrate the progressive substitution of polar atoms of the apoprotein by water molecules within the coordination sphere of the chloride anion, we have 
depicted a schematic free energy level diagram for the dynamic equilibrium between the different species involved in the process (see Fig. 9). The scheme is inspired in that one developed by Nandi and Bagchi ${ }^{4}$ concerning the bimodal dynamic behaviour of biological water. Their system presents just two states: free and bound water to a biomolecule. In our case, with the addition of a chloride anion, the scenario is more complex in the sense that the number of states, denoted as $\mathrm{Clw}_{i} \mathrm{p}_{j}$ in Fig. 9, increases significantly as well as the number of the different free energies involved ( $\Delta \mathrm{G}^{0}$ and $\Delta \mathrm{G}^{*}$ in Fig. 9). In addition, each state corresponds to an ensemble of species that may differ in the specific hydrogen bonds made with protein atoms.

As explained in the introduction, clarifying the binding/unbinding mechanism of anions such as chloride to cofactor binding pockets of apoflavodoxins bears on the understanding of the mechanism of FMN binding to this protein family and possibly to other FMN binding proteins. In both the Anabaena and $H p$ apoflavodoxins anions from the solvent replace the phosphate group of the FMN cofactor and contribute to stabilize the folded conformation of the protein. ${ }^{47,48}$ However, their presence poses a problem for the cofactor to bind as it would have first to displace the bound anion. Our mutational analyses of the transition state of FMN binding to either of those apoflavodoxins showed that the FMN cofactor first contact the apoprotein using its isoalloxazine aromatic moiety and only afterwards the phosphate moiety binds. ${ }^{36,37}$ Our present MD statistical analysis suggests one possible binding scenario of FMN binding to apoflavodoxins where the FMN cofactor partly bound to the apoprotein through the isoalloxazine moiety simply waits till whichever anion previously bound to the phosphate binding site gets released following the mechanism here described. Then, the FMN phosphate replaces the vacant left by the anion either immediately or after displacing the water molecules that, as seen in this study, can readily fill the vacant positions left by the leaving anion. Although this binding scenario is consistent with all 
the available data, an alternative scenario where the negatively charged phosphate moiety in FMN destabilizes the bound anion once the initial FMN/apoflavodoxin encounter complex takes place cannot be ruled out.

\section{CONCLUSION}

Analyses of the interaction between $H p$ apoflavodoxin, a bound chloride anion and solvent water molecules are consistent with a spontaneous release of the anion and a step-by-step mechanism in which protein/chloride interactions progressively break down before new water molecules gradually occupy the vacant positions left by protein atoms. Likewise, an alternative concerted mechanism in which water molecules play an active role forcing displacement of the protein atoms bound to the anion can be discarded. The reverse binding process would take place, therefore, when a chloride anion appearing in the bulk close to the FMN phosphate binding site opportunistically takes advantage of the breaking of protein/water interactions.

Acknowledgements. JS would like to acknowledge financial support from grants BFU201016297 (Ministerio de Ciencia e Innovación, Spain), BFU2013-47064-P and BIO2014-57314REDT (Ministerio de Economía y Competitividad, Spain), and PI078/08 (Gobierno de Aragón, Spain). MCM also acknowledge financial support provided by Grant No. FIS2014-55867-P (Ministerio de Economía y Competitividad, Spain) co-financed by FEDER funds, and from Grant No. E19 (Gobierno de Aragón, Spain). JJGF would like to acknowledge the Santander Bank and the University of Zaragoza (Spain) for having awarded him with a Ph.D. grant. 
Table 1. Number of chloride H-bonds before and after chloride release from the protein.

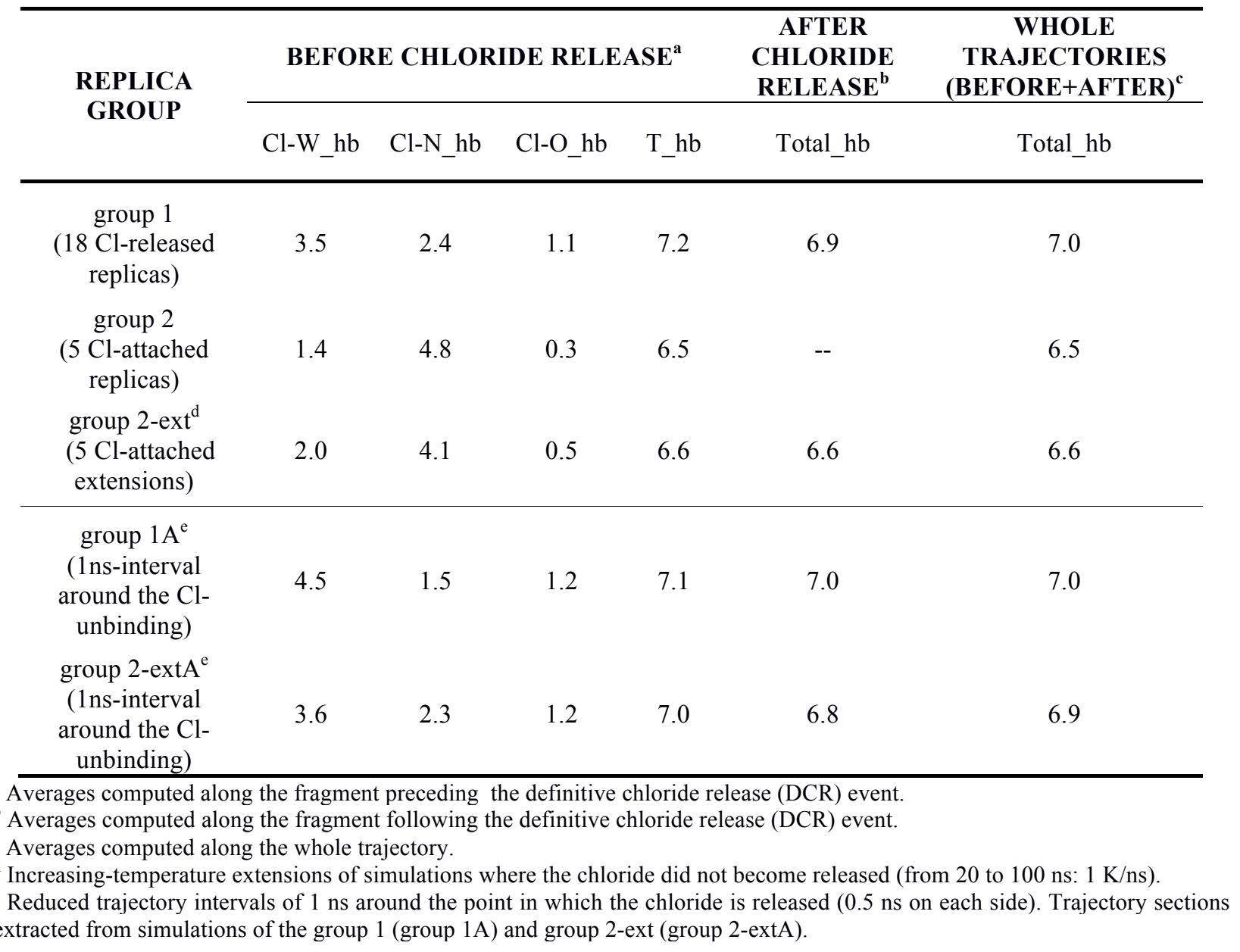




\section{REFERENCES}

1 S. A. Kuby, A Study of Enzymes: Enzyme Catalysis, Kinetics, and Substrate Binding, CRC Press, Boca Raton, FL., 1991.

2 L. Wang, X. Yu, P. Hu, S. Broyde and Y. Zhang, J. Am. Chem. Soc., 2007, 129, 47314737.

3 C. K. Brooks, J. J. Fawcett and J. Gittins, Nature, 1976, 260, 694-696.

$4 \quad$ N. Nandi and B. Bagchi, J. Phys. Chem. B 1997, 101, 10954-10961.

5 K. Bhattacharyya, Chem. Commun., 2008, 2848-2857.

6 M. C. Morón, Phys. Chem. Chem. Phys., 2012, 14, 15393-15399.

7 B. Bagchi and B. Jana, Chem. Soc. Rev., 2010, 39, 1936-1954.

8 B. Bagchi, Chem. Phys. Lett., 2012, 529, 1-9.

9 T. M. Raschke, Curr. Opin. Struct. Biol., 2006, 16, 152-159.

10 K. E. Furse and S. A. Corcelli, J. Phys. Chem. Lett., 2010, 1, 1813-1820.

11 M. Vogel, J. Phys. Chem. , 2009, 113, 9386-9392.

12 S. Kaieda and B. Halle, J. Phys. Chem. B 2013, 117, 14676-14687.

13 B. Bagchi, Chem. Rev., 2005, 105, 3197-3219.

14 S. K. Pal, J. Peon, B. Bagchi and A. H. Zewail, J. Phys. Chem. B, 2002, 106, 1237612395.

15 K. Sahu, S. K. Mondal, S. Ghosh and K. Bhattacharyya, Bull. Chem. Soc. Jpn., 2007, 80, 1033-1043.

16 I. D. J. Kuntz and W. Kauzmann, Adv. Protein. Chem. , 1974, 28, 239-345.

17 A. C. Pan, D. W. Borhani, R. O. Dror and D. E. Shaw, Drug Discovery Today, 2013, 18, 667--673.

18 B. Jayaram and T. Jain, Annu. Rev. Biophys. Biomol. Struct., 2004, 33, 343-361.

19 L. Jiang, B. Kuhlman, T. Kortemme and D. Baker, Proteins: Structure, Function, and Bioinformatics, 2005, 58, 893-904.

20 K. Yamasaki, T. Akiba, T. Yamasaki and K. Harata, Nucleic Acids Res., 2007, 35, 5073-5084.

21 M. S. Cheung, A. E. García and J. N. Onuchic, Proc. Natl. Acad. Sc.i U.S. A., 2002, 99, 685-690. 
22 T. Head-Gordon and S. Brown, Curr. Opin. Struct. Biol., 2003, 13, 160-167.

23 H. Kaya and H. S. Chan, J. Mol. Biol. , 2003, 326, 911-931.

24 A. Van der Vaart, B. D. Bursulaya, C. L. Brooks and J. K. M. Merz, J. Phys. Chem. B, 2000, 104, 9554-9563.

25 A. Migliore, S. Corni, R. Di Felice and E. Molinari, J. Phys. Chem. B, 2007, 111, 3774-3781.

26 R. Friedman, S. Fischer, E. Nachliel, S. Scheiner and M. Gutman, J. Phys. Chem. B., 2007, 111, 6059-6070.

27 X. Arias-Moreno, O. Abian, S. Vega, J. Sancho and A. Velazquez-Campoy, Current Protein and Peptide Science, 2011, 12, 325-338.

28 H. R. Faber, C. J. Baker, C. L. Day, J. W. Tweedie and E. N. Baker, Biochemistry, 1996, 35, 14473-14479.

29 D. Madern and C. Ebel, Biochimie, 2007, 89, 981-987.

30 A. A. Raibekas, E. J. Bures, C. C. Siska, T. Kohno, R. F. Latypov and B. A. Kerwin, Biochemistry, 2005, 44, 9871-9879.

31 C. H. I. Ramos and R. L. Baldwin, Protein Science, 2002, 11, 1771-1778.

32 O. Zak, K. Ikuta and P. Aisen, Biochemistry, 2002, 41, 7416-7423.

33 X. Tadeo, B. López-Méndez, D. Castaño, T. Trigueros and O. Millet, Biophysical J., 2009, 97, 2595-2603.

34 R. L. Baldwin, Biophys. J., 1996, 71, 2056-2063.

35 N. M. Antikainen and S. F. Martin, Bioorganic \& Medicinal Chemistry, 2005, 13, 2701-2716.

36 S. Ayuso-Tejedor, O. Abian, A. Velazquez-Campoy and J. Sancho, Biochemistry, 2011, 50, 8703-8711.

37 A. Lostao, F. Daoudi, M. P. Irun, A. Ramon, C. Fernandez-Cabrera, A. Romero and J. Sancho, J. Biol. Chem., 2003, 278, 24053-24061.

38 A. Lostao, C. Gómez-Moreno, S. G. Mayhew and J. Sancho, Biochemistry, 1997, 36, 14334-14344.

39 I. Nogués, L. A. Campos, J. Sancho, C. Gómez-Moreno, S. G. Mayhew and M. Medina, Biochemistry, 2004, 43, 15111-15121. 
40 A. Mitsutake, Y. Mori and Y. Okamoto, Methods Mol. Biol., 2013, 924, 153-195.

41 L. Monticelli and E. Salonen, eds., Biomolecular Simulations: Methods and Protocols, Methods in Molecular Biology, Springer Science+Business Media, New York, 2013.

42 C. D. Christ, A. E. Mark and W. F. van Gunsteren, J. Comput. Chem., 2010, 31, 15691582 .

43 A. de Ruiter and C. Oostenbrink, Curr. Opin. Chem. Biol., 2011, 15, 547-552.

44 N. Hansen and W. F. van Gunsteren, J. Chem. Theory Comput., 2014, 10, 2632-2647.

45 N. Cremades, M. Bueno, J. L. Neira, A. Velazquez-Campoy and J. Sancho, J Biol Chem, 2008, 283, 2883-2895.

46 N. Cremades, A. Velazquez-Campoy, E. Freire and J. Sancho, Biochemistry, 2008, 47, 627-639.

47 G. C. Genzor, A. Perales-Alcon, J. Sancho and A. Romero, Nat. Struct. Biol., 1996, 3, 329-332.

48 M. Martinez-Julvez, N. Cremades, M. Bueno, I. Perez-Dorado, C. Maya, S. CuestaLopez, D. Prada, F. Falo, J. A. Hermoso and J. Sancho, Proteins, 2007, 69, 581-594.

49 S. Ayuso-Tejedor, V. E. Angarica, M. Bueno, L. A. Campos, O. Abian, P. Bernado, J. Sancho and M. A. Jimenez, J Mol Biol, 2010, 400, 922-934.

50 S. Ayuso-Tejedor, R. Garcia-Fandino, M. Orozco, J. Sancho and P. Bernado, J Mol Biol, 2011, 406, 604-619.

51 N. Cremades and J. Sancho, Biophys J, 2008, 95, 1913-1927.

52 N. Cremades, J. Sancho and E. Freire, Trends Biochem Sci, 2006, 31, 494-496.

53 N. Cremades, M. Bueno, M. Toja and J. Sancho, Biophys. Chem., 2005, 115, 267-276.

54 N. Cremades, A. Velázquez-Campoy, M. Martínez-Júlvez, J. L. Neira, I. PérezDorado, J. Hermoso, P. Jiménez, A. Lanas, P. S. Hoffman and J. Sancho, ACS Chem. Biol., 2009, 4, 928-938.

55 J. J. Galano, M. Alias, R. Perez, A. Velazquez-Campoy, P. S. Hoffman and J. Sancho, J Med Chem, 2013, 56, 6248-6258.

56 J. J. Galano and J. Sancho, Med. Chem., 2013, 4, 306-312.

57 J. Freigang, K. Diederichs, K. P. Schafer, W. Welte and R. Paul, Protein Sci., 2002, 11, 253-261. 
58 W. Humphrey, A. Dalke and K. Schulten, J. Mol. Graph., 1996, 14, 33-38.

59 M. Rostkowski, M. H. M. Olsson, C. R. Sondergaard and J. H. Jensen, BMC Structural Biology, 2011, 11, 6.

60 J. C. Phillips, R. Braun, W. Wang, J. Gumbart, E. Tajkhorshid, E. Villa, C. Chipot, R. D. Skeel, L. Kale and K. Schulten, J Comput Chem, 2005, 26, 1781-1802.

61 S. E. Feller, Y. Zhang, R. W. Pastor and B. R. Brooks, J. Chem. Phys. , 1995, 103, 4613-4621.

62 A. D. MacKerell, D. Bashford, M. Bellot, R. L. Dunbrack, J. D. Evanseck, M. J. Field, S. Fischer, J. Gao, H. Guo, S. Ha, D. Joseph-McCarthy, L. Kuchnir, K. Kuczera, F. T. K. Lau, C. Mattos, S. Michnick, T. Ngo, D. T. Nguyen, B. Prodhom, W. E. Rehir, B. Roux, M. Schlenkrich, J. C. Smith, R. Stote, J. Straub, M. Watanabe, J. WirkiewiczKuczera, D. Yin and M. Karplus, J. Phys. Chem. B, 1998, 102, 3586-3616.

63 G. J. Martyna, D. J. Tobias and M. L. Klein, J. Chem. Phys., 1994, 101, 4177-4189.

64 U. Essmann, L. Perera, M. Berkowitz, T. Darden, H. Lee and L. Pedersen, J. Chem. Phys. , 1995, 103, 8577-8593.

65 E. Arunan, G. R. Desiraju, R. A. Klein, J. Sadlej, S. Scheiner, I. Alkorta, D. C. Clary, R. H. Crabtree, J. J. Dannenberg, P. Hobza, H. G. Kjaergaard, A. C. Legon, B. Mennucci and D. J. Nesbitt, Definition of the hydrogen bond. In Recommendation submitted by the IUPAC task group (2004-026-2-3 100), IUPAC, http://media.iupac.org/reports/provisional/abstract11/arunan_prs.pdf, 2004.

66 S. Busch, L. C. Pardo, W. B. O'Dell, C. D. Bruce, C. D. Lorenz and S. E. McLain, Phys. Chem. Chem. Phys., 2013, 15, 21023-21033.

67 L. Ge, L. Bernasconi and P. Hunt, Phys. Chem. Chem. Phys., 2013, 15, 13169-13183.

68 T. Ida, M. Ando and H. Toraya, Journal of Applied Crystallography, 2000, 33, 13111316 .

69 L. Aaron, E. Stancik and B. Brauns, Vibrational Spectroscopy, 2008, 47, 66-69.

70 R. Friedman, E. Nachliel and M. Gutman, Biophys. J., 2005, 89, 768-781.

71 G. A. Jeffrey, ed., An introduction to hydrogen bonding, Oxford University Press, 1997. 
72 S. J. Grabowski, ed., Hydrogen Bonding-New Insights, Springer, Dordrecht, The Netherlands, 2006. 


\section{FIGURES}

Fig. 1. Structural alignment of holo and apo HpFld. Cartoons of the holo Hp-Fld (PDB code: 1FUE, in green) showing the FMN cofactor (in sticks); the apo $H p$-Fld (PDB code: $2 \mathrm{BMV}$, in yellow) displaying the chloride (green sphere) mimicking the phosphate group of the FMN; and the apo Fld from Anabaena (PDB code: 1 FTG, in blue) displaying a sulphate anion (in sticks) at the same position.

Fig. 2. Radial distribution functions. Radial distribution functions, $g(r)$, for both chloride/water and chloride/sub-pocket-1 pairs from MD simulations. Among the chloride/sub-pocket-1 pairs, plots are shown for each of the H-bond donor atoms from this sub-pocket, namely, backbone nitrogens (N) of residues THR10, ASP11, SER12, GLY13, ASN14 and ALA15, the sidechain nitrogen (ND) of ASN14, as well as sidechain oxygens (OG) of THR10 and SER12. Plots were obtained by averaging the results collected for ten replicas randomly chosen from the fifty simulations initially performed.

Fig. 3. Coordination profiles of the chloride in the binding pocket where the phosphate group of the FMN cofactor binds. A) A typical coordination pattern with the respective H-bond distances observed during one representative simulation where the chloride did not release; about 5 nitrogen atoms appear well-bonded around the anion (H-bond distances $<3.0 \AA$ ). B) Weakened coordination profile mostly observed along replicas where the chloride did release soon; about 3 or less well-bonded nitrogen atoms appear coordinating the chloride. C) Chloride/protein coordination profile and the respective $\mathrm{H}$-bond distances found in the apo $\mathrm{Hp}$-Fld crystal structure (PDB code: 2BMV). The green sphere represents the chloride anion, whilst residues of the sub-pocket are showed in sticks.

Fig. 4. Chloride exit routes and chloride release times from the protein. A) Apo $H p$-Fld cartoon showing (in sticks) the three sub-pockets constituting the overall FMN binding pocket and three possible exit routes of the chloride. B) Bars diagram showing the replicas percentages in which the chloride exits by the indicated routes. Sub-pocket 1 (cyan): residues THR10, ASP11, SER12, GLY13, ASN14 and ALA15; sub-pocket 2 (yellow): PRO53, THR54, ALA55, GLY56 and GLN61; sub-pocket 3 (red): GLY87, ASP88, ASP140, GLU141 and ASP142. C) Number of 
simulations, out of the fifty initially performed, as a function of time interval in which the chloride release is produced from the FMN phosphate binding site (sub-pocket 1, see Fig. 3). Blue bars refer to 45 out of the 50 replicas where chloride is released either during the 20nsproduction stage (time from 0 to $20 \mathrm{~ns}$ ) or before (negative times). Red bars represent the remaining 5 replicas where chloride unbinds during the extended phase (100 ns) in which the temperature is increased at a rate of $1 \mathrm{~K} / \mathrm{ns}$ up to an average temperature of $357 \mathrm{~K}$. The inset is a zoomed histogram that provides further details on release times.

Fig. 5. Coordination and distance analysis. A) Evolution of the chloride/water (red line), chloride/protein (blue line) H-bonds, and the sum of both (yellow line), along a time window in which the chloride release event is observed. Orange lines are the result of the superposition of yellow and red colour lines. B) Time dependence of the distances to the chloride anion showing plots of the last polar atoms of the protein that are released and of one water molecule entering the first solvation shell of the anion (see legend). The FIDCR and FIWE moments are indicated. A1, B1) Zoomed sections around the chloride unbinding moment. Graphs are obtained from one representative out of the ten performed high-resolution simulations.

Fig. 6. Histograms of water entry (WE) events relative to the superimposed time $\left(t_{0}\right)$ where polar atoms of the protein are released from the chloride (PAR events). A) WEs registered along 50 frames around $t_{0}$ (25 on each side). B) Grouped or 'compacted' histogram of WEs where each bar represents the events registered in 5 consecutive frames of time.

Fig. 7. 'Compacted' histograms and fitting function for WE events obtained using different FIPAR cutoff distances. Histograms depict WEs of 5 consecutive frames within the interval 3.3$3.7 \AA$. All histograms show the maximum number of water entries (WE events) slightly shifted to the right of $t_{0}$ (FIPAR event). The distribution has been fitted to an asymmetric pseudo-Voigt function (see section IIe). These results show that the analyses performed using FIPAR cutoffs within that range do not affect the conclusion that WE events mostly occur once PARs $\left(t_{0}\right)$ have taken place.

Fig. 8. Alternative mechanisms for the chloride unbinding process. On the top, a hypothetical concerted mechanism where water would play the key role by strongly solvating the chloride - 
through an intermediate exceeding the natural valence of the anion- and snatching it from its binding site. On the bottom, a 'step-by-step' mechanism where protein/chloride H-bonds are first broken and afterwards new water molecules gradually occupy the valences left by the protein.

Fig. 9. Schematic free energy diagram showing the dynamic equilibrium $\left[\mathrm{Clw}_{i} \mathrm{p}_{j}\right] \Leftrightarrow\left[\mathrm{Clw}_{i+l} \mathrm{p}_{j-1}\right]$ between different species corresponding to the chloride bound to the apoprotein and to solvent molecules. The acronym $\mathrm{Clw}_{i} \mathrm{p}_{j}$ represents the anion hydrogen bonded to $i$ water molecules and $j$ polar atoms of the biomolecule while $\mathrm{Clw}_{i} \mathrm{p}_{j-1}$ stands for the transition state. $\Delta \mathrm{G}^{0}$ shows the difference in free energy of a hydrogen bond between the chloride and a water molecule, and, between the chloride and the protein (states $\mathrm{Clw}_{i} \mathrm{p}_{j}$ and $\mathrm{Clw}_{i+1} \mathrm{p}_{j-1}$ ) while $\Delta \mathrm{G}^{*}$ denotes the activation energy of chloride desorption from the surface of the apoprotein (states $\mathrm{Clw}_{i} \mathrm{p}_{j}$ and $\left.\mathrm{Clw}_{i} \mathrm{p}_{j-1}\right)$. 
Fig. 1

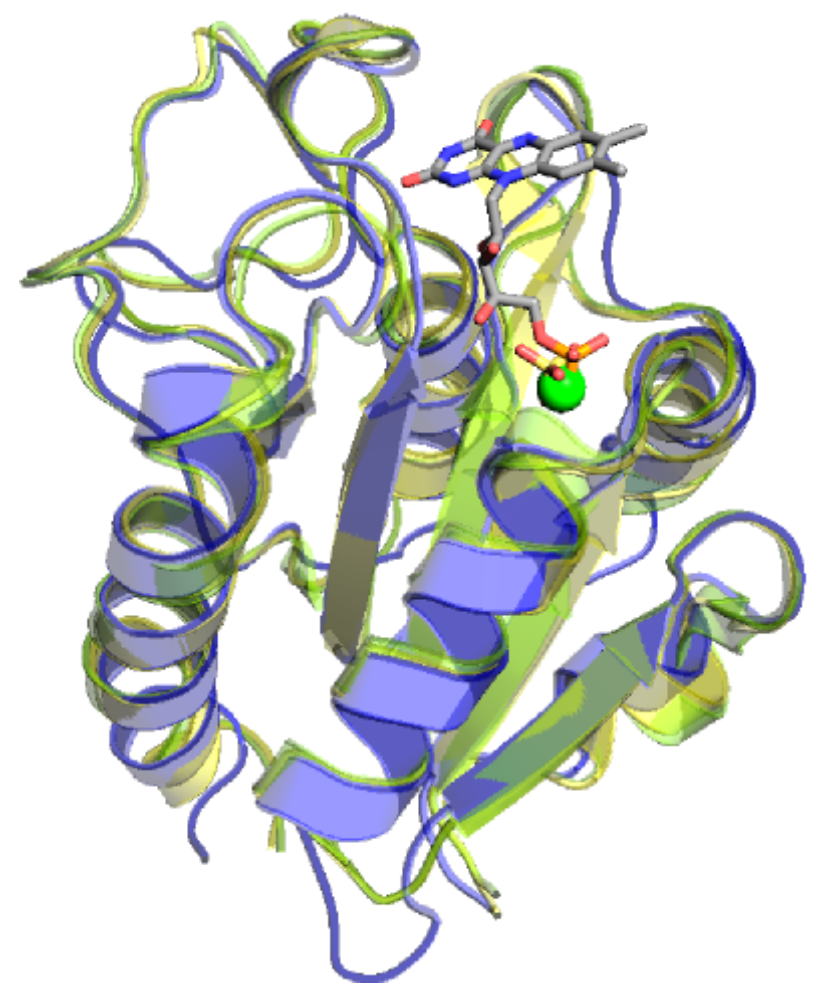


Fig. 2
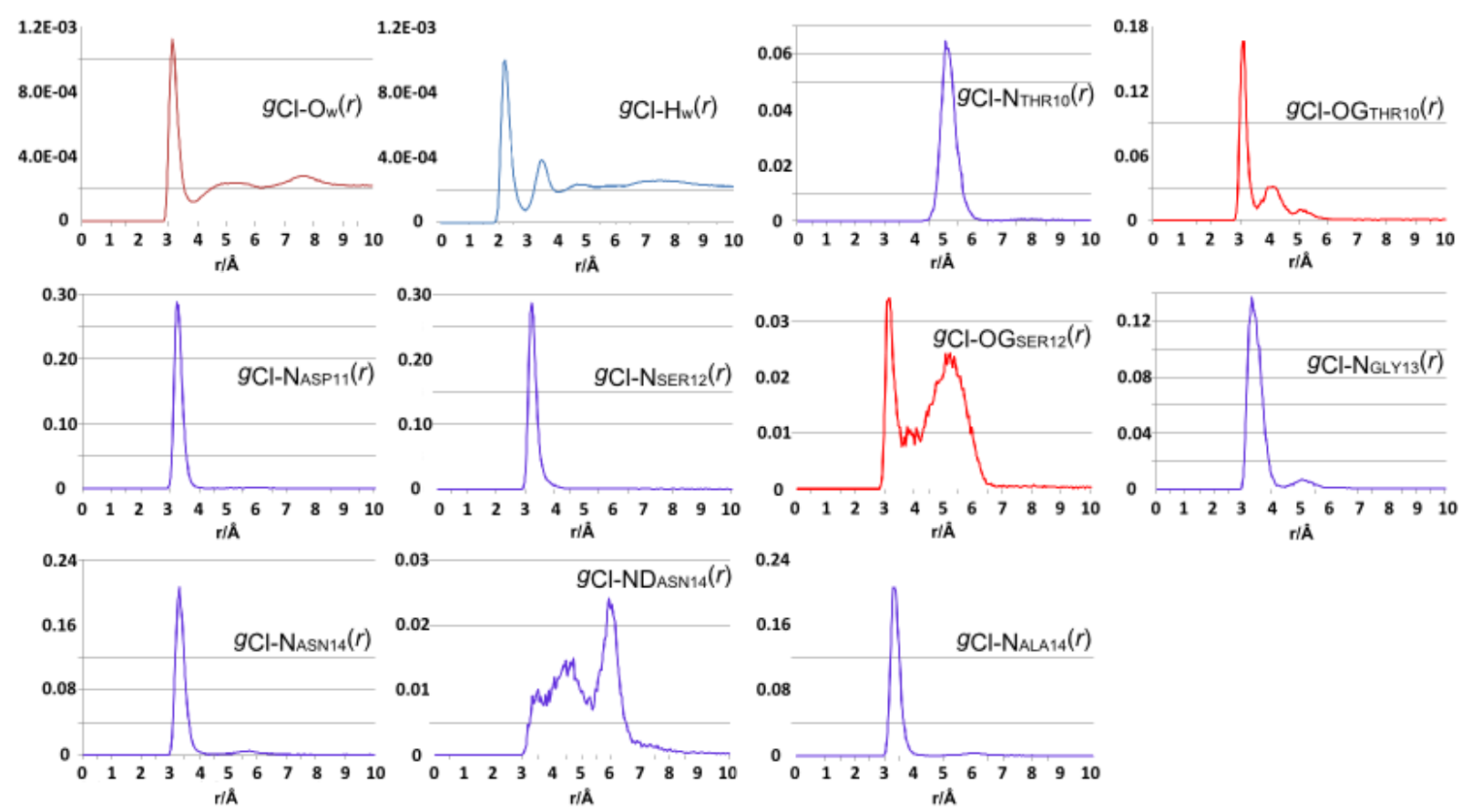
Fig. 3
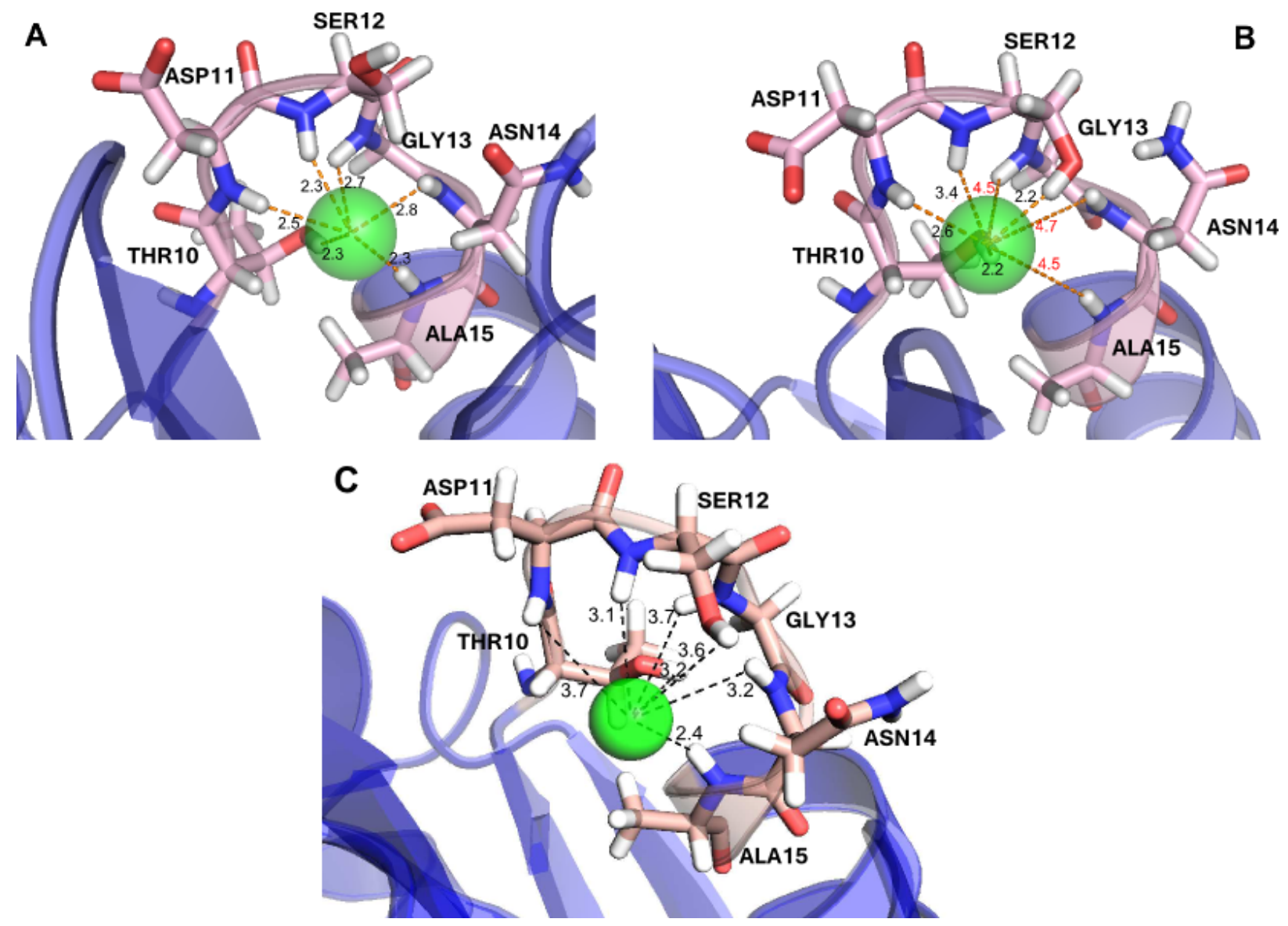
Fig. 4

A

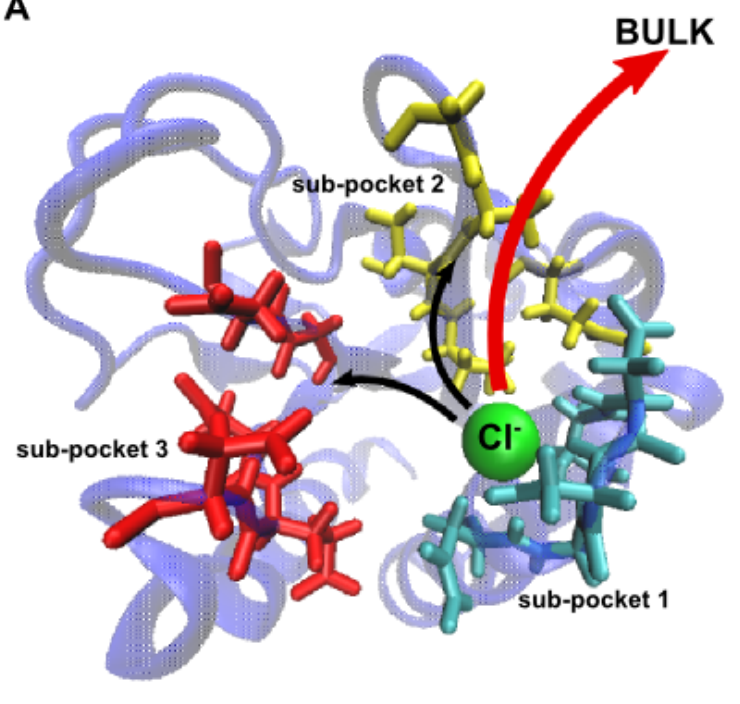

B

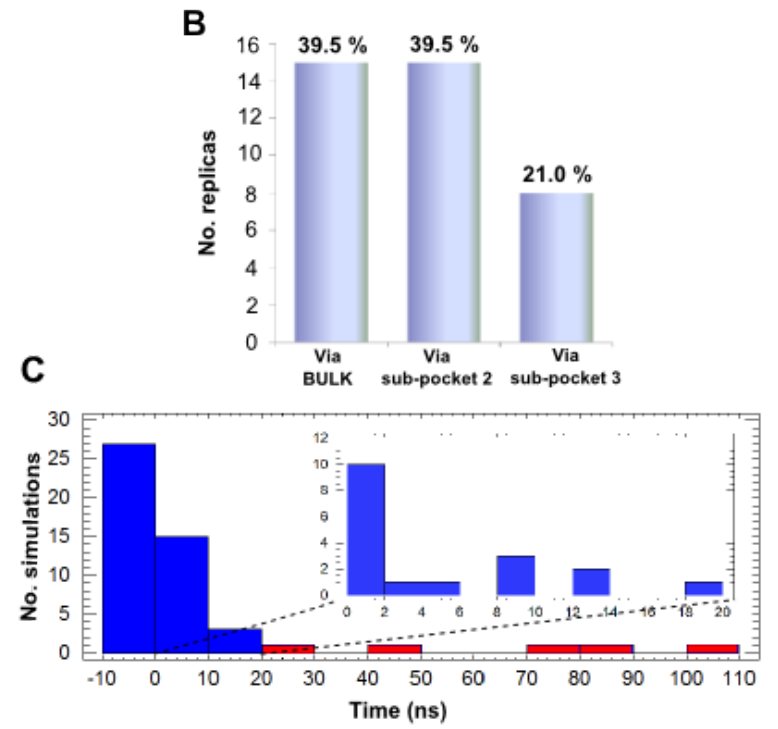

